\title{
Vingt ans au cirque. Des « Roman circuses » au «Cirque romain »
}

Jean-Paul Thuillier

\section{(2) OpenEdition}

1 Journals

Édition électronique

URL : http://journals.openedition.org/edl/125

DOI : $10.4000 /$ edl. 125

ISSN : 2296-5084

Éditeur

Université de Lausanne

\section{Édition imprimée}

Date de publication : 15 mai 2011

Pagination : $325-340$

ISBN : 978-2-940331-25-3

ISSN : 0014-2026

\section{Référence électronique}

Jean-Paul Thuillier, «Vingt ans au cirque. Des « Roman circuses » au « Cirque romain » », Études de lettres [En ligne], 1-2 | 2011, mis en ligne le 15 mai 2014, consulté le 18 décembre 2020. URL : http:// journals.openedition.org/edl/125; DOI : https://doi.org/10.4000/edl.125 


\section{VINGT ANS AU CIRQUE DES «ROMAN CIRCUSES » AU "CIRQUE ROMAIN »}

Vingt ans après la sortie, en 1986, du livre phare de John Humphrey sur les cirques romains, l'organisation d'un colloque international à Bordeaux a permis de faire le point sur toutes les nouveautés concernant cet édifice de spectacle aux dimensions impressionnantes. De l'ouvrage publié en 2008 et qui réunit beaucoup d'articles portant sur les spectacles eux-mêmes, sur leur organisation et leur réception, ne sont examinées ici que les études proprement archéologiques: mais il suffira de mentionner la découverte récente d'un cirque romain en Angleterre pour apprécier tout l'intérêt de cette première partie.

Le cirque n'est pas l'édifice romain de spectacle le plus étudié. De nombreuses raisons d'ordre divers concourent à cet état de choses. Il s'agit tout d'abord d'un monument lié au sport, puisque le circus était dédié essentiellement aux compétitions hippiques et athlétiques, et pendant longtemps ce thème n'a pas été considéré comme un sujet noble, susceptible de mériter l'attention des universitaires, des chercheurs; certes, la situation a beaucoup évolué dans ces dernières années, et l'existence d'une revue d'histoire du sport antique reconnue comme Nikephoros en apporte un témoignage significatif, mais il reste que le théâtre antique par exemple, et les ludi scaenici, par leur dimension plus intellectuelle, suscitent un volume d'analyses et de recherches qui ne saurait être comparé à celui qu'engendrent le circus et les ludi circenses. Pour prolonger cette question, on a même fait remarquer, avec un peu d'humour, à propos du théâtre et des pièces dramatiques, que les études sur la tragédie seraient mieux considérées universitairement que les études sur la comédie: dans la hiérarchie des recherches, le sérieux de l'objet sur lequel on travaille rejaillirait inévitablement sur l'étude qui est menée... et 
donc sur le chercheur lui-même. Ainsi travailler sur le sport ne pourrait être le fait que d'aimables «amateurs "...

Mais, après ce petit mouvement de mauvaise humeur, venons-en à des faits plus objectifs qui expliquent cette situation. D'une part, il y a beaucoup moins de cirques dans le monde romain qu'il n'y a de théâtres et d'amphithéâtres; on ne peut en citer qu'un peu plus d'une cinquantaine. Ainsi, on peut dire que l'édifice destiné à accueillir les spectacles sportifs, hippiques, mais aussi athlétiques, ne fait pas pleinement partie de la panoplie monumentale des villes romaines: une affirmation qui doit cependant être corrigée lorsqu'il s'agit des capitales, des mégapoles de l'Empire romain, comme le montrent suffisamment les exemples de Rome elle-même, de Carthage, de Tarragone, de Lyon, pour ne pas parler de Constantinople.

D'autre part, le circus est infiniment plus grand que les deux autres édifices auxquels nous venons de faire allusion : ce qui explique précisément qu'on ne pouvait en construire en dur dans toutes les villes, grandes et moyennes. La figure $1 \mathrm{du}$ livre de John Humphrey ${ }^{1}$, sur lequel nous allons tout de suite revenir, est très parlante de ce point de vue, puisqu'on peut constater que l'arène du Colisée rentrerait douze fois dans celle du Circus Maximus! Dans ces conditions, la fouille d'un cirque est une entreprise colossale: il faut évidemment se limiter à des sondages pertinents, à supposer que les zones visées n'aient pas été détruites ou bouleversées par l'urbanisme galopant ou par une tout autre raison. Reste de toute façon ensuite à mener à bien la publication d'un tel édifice, une entreprise qui n'est évidemment pas facilitée par sa grandeur et sa complexité.

Signalons enfin qu'on a parfois tendance à réduire curieusement l'originalité du cirque en tant que monument de spectacle romain. On insiste depuis longtemps sur le fait que l'amphithéâtre serait la seule vraie création romaine, les trois autres édifices, théâtre, cirque, stade, n'étant que des avatars de leurs correspondants grecs. Dans son excellent livre sur l'amphithéâtre romain des origines au Colisée, publié très récemment, Katherine E. Welch revient encore sur cette idée:

Indeed, the Roman theatre was derived from the Greek theatre, the Roman stadium from the Greek stadium, and the Roman circus from

I. J. H. Humphrey, Roman circuses. 
the Greek hippodrome. The amphitheatre, on the other hand, had no obvious Greek architectural precedent ${ }^{2}$.

Et elle poursuit cette thèse à propos des spectacles eux-mêmes: les munera seraient les seuls à n'avoir pas de précédent hellénique. Sans insister sur le fait que, d'un point de vue lexical, contrairement au stade et au théâtre, le mot circus n'est pas en relation avec le mot hippodrome, une telle présentation fait bon marché de la très longue histoire du cirque et des jeux du cirque qui remontent au VII siècle avant notre ère (sans parler de Romulus ni de l'enlèvement des Sabines), sous le règne de Tarquin l'Ancien; et c'est oublier justement que ce ne sont pas les influences grecques, mais les influences étrusques qui ont été prépondérantes en ce domaine (techniques et tenue de l'aurige, courses de triges, Trigarium, absence de courses de chevaux montés, etc.). Quant à l'édifice circus considéré d'un point de vue architectural, il est bien autre chose que l'hippodrome grec qui n'a jamais présenté l'aspect d'un édifice complètement construit, même si celui d'Olympie, d'après Pausanias et quelques vestiges mis au jour, comportait certaines structures en dur. En tout cas, on ne peut pas réduire la différence entre les deux à de simples aménagements: la seule existence d'un mur-barrière (la cosidetta spina) est révélatrice. Il est donc tout à fait erroné de parler du cirque romain comme d'un simple avatar de l'hippodrome grec, et il est au contraire nécessaire d'insister sur son caractère décidément italique.

Après ce bref rappel, la sortie en 1986 du livre de John Humphrey sur les Roman circuses. Arenas for chariot racing a constitué un véritable événement pour les spécialistes de la question. Si son espoir de voir ensuite apparaitre des monographies sur différents cirques a pu être un peu déçu, il faut saluer l'heureuse initiative qu'a été l'organisation d'un grand colloque international à Bordeaux sur ce thème en 2006, c'est-à-dire exactement vingt ans après la publication de l'ouvrage de John Humphrey. Initiative due à un groupe très actif de l'Institut Ausonius de l'Université de Bordeaux 3 - on se contentera de citer ici les noms de Jean-Claude Golvin, de Jocelyne Nelis-Clément, de Jean-Michel Roddaz et de Robert Vergnieux - dont le travail ne s'est pas limité à ce colloque (ce qui ne serait déjà pas mal!). En effet, les années précédentes ont vu la mise au

2. K. E. Welch, The Roman amphitheatre, p. 162. 
point d'une maquette numérique du Circus Maximus et l'organisation de plusieurs séminaires destinés à préciser divers éléments de l'édifice trop longtemps négligés, sans parler de la réunion d'une documentation littéraire et figurée presque exhaustive: ainsi, le colloque ne faisait-il que couronner une entreprise de longue haleine, sans qu'il n'y ait d'ailleurs une quelconque prétention à régler tous les problèmes, la maquette pouvant évoluer en fonction de découvertes, de données et d'études nouvelles. On ne peut que saluer ce travail pleinement pluridisciplinaire qui a donc fait intervenir des historiens, des archéologues, des architectes et des informaticiens.

Ce sont donc des actes de ce colloque, publiés sous le titre Le cirque romain et son image, dont il va être question ici. Le livre de John Humphrey était surtout archéologique, comme il l'indiquait lui-même dans sa préface avec une certaine modestie: pour bien comprendre les courses hippiques romaines, il fallait bien connaître l'édifice dans lequel elles se déroulaient - et on pourrait ajouter aujourd'hui, du point de vue méthodologique, que l'inverse est aussi vrai, comme l'établissement de la maquette électronique l'a montré à chaque étape. A vrai dire, l'ouvrage de John Humphrey comporte un grand nombre d'informations sur les ludi circenses, en particulier dans les notes très abondantes et très développées. Et c'est évidemment ce parti qu’ont adopté depuis l'origine les responsables bordelais de l'opération Circus Maximus et c'est ce parti que l'on retrouve dans le livre publié en 2008, qui comprend donc une partie sur les édifices ("le modèle archéologique») et une partie sur les spectacles offerts dans ces édifices - les deux parties étant de longueur à peu près égale. Même si nous aussi nous mettrons l'accent dans ce compte rendu presque uniquement sur la partie archéologique, insistons sur le fait que cette double approche était absolument nécessaire car la spécialisation est si poussée aujourd'hui qu'elle permet rarement d'avoir une vue synthétique: cela est vrai même quand il s'agit d'un thème très précis et l'on constate d'ailleurs dans cet ouvrage qu'il peut y avoir des distorsions étonnantes entre les "archéologues" et les "historiens/littéraires" pour utiliser ces termes imprécis mais commodes: les bibliographies propres à chaque étude sont très révélatrices de ce point de vue.

On est également surpris de constater que certaines remarques décisives faites par John Humphrey en 1986 ne sont toujours pas prises en compte: c'est ainsi que beaucoup d'auteurs continuent à employer sans sourciller le mot spina pour désigner le mur-barrière axial, alors qu'il 
était désigné en latin sous le nom d'euripus - c'est seulement au VI ${ }^{\mathrm{e}}$ siècle de notre ère, chez Cassiodore, qu'on rencontre le terme de spina. On comprend bien qu'il y ait quelque difficulté à utiliser le mot "euripe" pour ce mur puisqu'il désignait aussi les fossés remplis d'eau que fit creuser César en 46 av. J.-C. autour de l'arène. Mais alors il faudrait au moins justifier le choix qui est fait sur le plan du lexique; sinon, on perpétue des erreurs anciennes et on fait même oublier les résultats scientifiques novateurs. De la même façon - mais ce n'est pas le cas dans ce livre - et pour prendre un exemple que j'ai souvent dénoncé, l'expression "les jeux du cirque" continue à être comprise par le grand public, par les journalistes et même par un grand nombre d'universitaires comme renvoyant aux combats de gladiateurs... qui ne se sont jamais ou presque jamais déroulés dans un cirque. Il est des lieux communs très difficiles à extirper et si les chercheurs ne se mettent pas de la partie, on n'a aucune chance d'arriver à un résultat positif.

A tout seigneur, tout honneur: c'est le Circus Maximus qui ouvre le volume et sa partie architecturale, avec une contribution de Paola Ciancio Rossetto qui a consacré au monument de nombreux articles, en particulier dans le Lexicon topographicum urbis Romae, et qui a été associée depuis longtemps à l'opération de la maquette électronique évolutive. Ce travail a rendu nécessaire une analyse archéologique soutenue, et l'on connaît bien les difficultés de la fouille dans cette zone, avec la présence de l'eau dans le sous-sol et l'installation de structures industrielles au début du siècle dernier (cf. la photo spectaculaire reproduite en fig. 2). Larchéologue italienne présente un résumé de l'histoire de l'édifice, avec par exemple l'importance des travaux réalisés sous Domitien, toujours intéressé par les édifices de spectacle, ainsi que sous Trajan. Les visiteurs qui connaissent Rome savent bien qu'il ne reste pas beaucoup de vestiges visibles de ce qui fut peut-être le plus grand édifice de spectacle de tous les temps: on voit surtout quelques éléments de l'hémicycle, où deux projets différents de construction ont été mis en œuvre; on n'a même pas retrouvé le mur de podium, mais les salles voûtées de l'ambulacre extérieur fonctionnent selon un rythme ternaire du côté du Palatin (taberna, passage vers le couloir intermédiaire, escalier), et l'on peut donc tenter de suivre dans cette zone les différents parcours du public (cf. fig. 17). L'article de Paola Ciancio Rossetto se termine sur une note optimiste: sont en effet prévus de grands projets destinés à protéger et à mettre en 
valeur le Grand Cirque dans son environnement urbain, et cela conduira à de nouvelles fouilles archéologiques prometteuses, puisque poussées en profondeur dans certains cas.

Cette étude synthétique est complétée par une intervention courte mais très suggestive de Maria Letizia Buonfiglio à propos des systèmes hydrauliques de l'édifice qui devaient effectivement être d'une grande complexité: tout en remarquant là encore une différence, parallèle à celle du bâti, entre les deux zones (Palatin/Aventin) de l'hémicycle, M. L. Buonfiglio signale l'existence et la situation de conduites verticales, de tubuli, et de latrines. En fait, cette question de l'eau est fondamentale tout au long de l'histoire de l'édifice:

La formazione della valle, i riti ed i culti arcaici, le corse e le varie manifestazioni, il monumento, e poi, successivamente, gli orti, i mulini e l'occupazione industriale con gli opifici del secolo XIX non sarebbero stati possibili senza la presenza e il passagio dell'acqua con il suo vario utilizzo ${ }^{3}$.

Qu'on songe simplement au grand égout qui, à l'époque des rois étrusques, devait traverser la vallée Murcia comme la Cloaca Maxima au Forum et l'on prendra conscience de nos ignorances actuelles (des notes très fournies nous mettent ici, si l'on peut dire, l'eau à la bouche). Quant à Jean-Claude Golvin, à qui l'on doit plusieurs interventions dans le volume, il revient d'abord sur un des problèmes les plus complexes du Circus Maximus, qui est celui de la situation du pulvinar, à la fois loge et sanctuaire: cet édifice s'est promené au cours des siècles avant de trouver sa place en haut de la cavea sous le règne de Trajan. Jean-Claude Golvin s'intéresse aussi à l'évolution de l'euripe et pose une question intéressante: avant que celui-ci comporte des bassins, c'est-à-dire sans doute avant Néron, où les sparsores puisaient-ils leur eau? Dans le fossé autour de la piste? A des fontaines? Ou avaient-ils une réserve d'amphores?

A l'inverse du Grand Cirque, le cirque de Maxence est remarquablement conservé, comme le montre bien la vue aérienne de la figure 3, dans l'article de Giuseppina Pisani Sartorio qui a déjà donné en 1999, avec Giovanni Ioppolo, une excellente monographie du monument ${ }^{4}$. Elle s'attache ici, dans un appendice, aux autres cirques de l'Italie dont

3. J. Nelis-Clément, J.-M. Roddaz (éds), Le cirque romain et son image, p. 45.

4. G. Ioppolo, G. Pisani Sartorio, La villa di Massenzio sulla Via Appia. 
on sait qu'ils n'étaient pas nombreux - et la pseudo-lettre de Mécène à Auguste chez Dion Cassius (52, 30, 7-8) en témoigne de façon révélatrice. Mais on peut trouver curieux que son catalogue associe le campus au cirque, alors qu'il s'agit de deux types d'édifices différents, même si des activités sportives s'y déroulaient: pourquoi d'ailleurs traiter le Trigarium de campus ${ }^{5}$ alors qu'il s'agit bien, depuis l'époque étrusque, d'un cirque, certes dédié surtout à l'entraînement, mais qui abritait aussi parfois des compétitions officielles? C'est le Campus Martius dans son ensemble qui était un campus et qui a d'ailleurs servi de modèle à tous les campi de province. Giuseppina Pisani Sartorio présente ensuite les diverses structures du cirque probablement construit après le mausolée et le palais: portes, oppidum, carceres, cavea reposant sur une voûte allégée par des amphores, pulvinar, tribune des juges.

Mais c'est surtout la décoration sculptée de l'euripe qui est étudiée de façon remarquable: la statue de Vénus ou de Nymphe à la coquille (fig. 36, dans la collection Torlonia, non accessible) serait-elle à mettre en rapport avec les sparsores qui sont parfois munis non d'une amphore mais d'un bassin (voir par exemple le bas-relief d'Ostie, certains gobelets de type Aco, la mosaïque de Lyon)? Mais il ne faut plus dire que les sparsores étaient là pour «répandre de l'eau sur la piste et les moyeux des roues de chars»: une tentative qui eût été bien dérisoire ${ }^{6}$. Leur fonction consistait en réalité à jeter de l'eau sur la bouche et les naseaux des chevaux qui auraient pu être étouffés par la chaleur et la poussière de l'arène ${ }^{7}$. Ces professionnels du cirque, qui devaient jouir d'une popularité non négligeable auprès du public, nous amènent à faire une rapide et exceptionnelle incursion vers la seconde partie de l'ouvrage de Bordeaux. Dans un article consacré à ce qu'il appelle très justement des "produits dérivés» du cirque ${ }^{8}$, Christian Landes cite une jolie fibule discoïde en alliage cuivreux qui porte le nom d'un cocher, Epaphroditus, et d'un cheval, Bubalus, déjà connus par d'autres documents épigraphiques 9 . Or, ce type de fibule ronde apparaît à plusieurs reprises dans la tenue de

5. J. Nelis-Clément, J.-M. Roddaz (éds), Le cirque romain et son image, p. 73.

6. Ibid., p. 63.

7. J.-P. Thuillier, "Agitator ou sparsor?»; ainsi que W. Decker, J.-P. Thuillier, Le sport dans l'Antiquité, p. 196-201.

8. J. Nelis-Clément, J.-M. Roddaz (éds), Le cirque romain et son image, p. 413-430.

9. Mais l'objet vient d'Eure-et-Loir, et non pas d'Indre-et-Loir: voir l'article récent de Y. Barat, V. Venet, «Epaphrodite et sa "gazelle”». 
sparsores (notamment sur le relief d'Ostie conservé au Vatican, les bustes en marbre du sanctuaire d'Hercules Cubans au Trastevere), et on aurait ainsi un autre exemple de ce lien souvent affirmé au sein d'une faction entre agitatores et sparsores.

On passe ensuite aux provinces et d'abord à l'Afrique qui fut une grande terre de cheval et de passion pour les courses hippiques, comme on le sait: Louis Maurin ne peut que constater l'absence de toute nouvelle découverte de cirque - et ainsi y a-t-il toujours la même disparité régionale, aucun cirque n'étant identifié en Tingitane. C'est bien sûr Carthage qui reste le morceau de choix, du fait de ses dimensions $(570 \mathrm{~m}$ sur 130 m environ, une capacité de presque 50000 spectateurs), et du fait que des fouilles ont pu y être menées dans ces dernières décennies ${ }^{10}$. Si le cas de Dougga, que connaît bien l'auteur, est traité à part en raison du caractère modeste de l'édifice, ce cirque est cependant très intéressant par son dossier épigraphique et par le fait qu'on a privilégié ici ce monument et non l'amphithéâtre, ce qui est exceptionnel dans ces petites cités africaines. Mais le graffito inscrit sur le stylobate du portique des Templa Concordiae (fig. 17) représente-t-il un cirque? On peut en douter: si la forme ovale peut trouver un parallèle dans la mosaïque de Carthage conservée au Bardo (fig. 18), les carceres seraient figurés au sommet de la cavea (?), et surtout il n'y a pas d'euripe (la cosidetta spina): or, c'est là l'élément le plus sûr d'identification d'un cirque ${ }^{11}$. L'Afrique toujours, mais cette fois dans l'Antiquité tardive, avec l'étude de Ridha Ghaddab qui déborde le simple cadre du cirque et qui présente des tableaux très complets sur les édifices de spectacle et les spectacles eux-mêmes dans les diverses provinces de l'Afrique. Les données réunies par l'auteur le conduisent à remettre en question un certain nombre de conclusions généralement admises: il minimise la diffusion des spectacles, insiste sur les difficultés financières des cités, rappelle qu'à l'époque vandale il n'y a guère que Carthage à garder cette pratique des spectacles publics qui en tout état de cause ne sont plus attestés sous la domination byzantine, la métropole africaine n'échappant plus à la règle; les édifices de spectacle servent alors de carrières pour la construction des citadelles et des murailles.

IO. A propos des nombreuses tabellae defixionis qui ont été mises au jour, en particulier dans l'arène du cirque, on citera maintenant J. Tremel, Magica agonistica.

II. Voir d'ailleurs le graffito de Belalis Maior, p. 105, n. 49. 
Hazel Dodge réexamine la question des cirques dans les provinces orientales de l'Empire, qui a soulevé quelques controverses sur la terminologie en particulier ${ }^{12}$. Il s'attarde surtout sur des découvertes récentes, et d'abord en Grèce même, une région qui a bien évidemment une longue tradition d'agônes hippiques. On peut d'ailleurs en profiter pour rappeler que l'hippodrome d'Olympie a peut-être bien été retrouvé lors de prospections géophysiques récentes, qui ont permis d'identifier des traces linéaires sur plus de $1000 \mathrm{~m}$ : de toute façon, ce serait dans la zone prévue depuis toujours, entre le stade et le cours de l'Alphée, et on avait déjà identifié un toit de tuiles correspondant au bloc de départ ${ }^{13}$. Il semble en tout cas qu'on puisse désormais parler d'un circus, situé de façon assez centrale, dans la colonie romaine de Corinthe (vestiges d'une meta et d'un tronçon de la cosidetta spina, p. 138 sq.), mais on peut espérer que des fouilles futures permettront d'en cerner les dimensions ${ }^{14}$.

Le cirque de Jerash, qui a été édifié dans la seconde moitié du $\mathrm{II}^{\mathrm{e}}$ siècle de notre ère, avait bien une barrière centrale, même si celle-ci est de faible longueur aujourd'hui. Quant au complexe hérodien de Caesarea Maritima, il se révèle comme un édifice flexible, jouant à la fois le rôle de cirque, d'amphithéâtre et de stade - un trait que l'on connaît dans d'autres édifices de spectacle de ces régions orientales. Il est bien sûr dans l'Orient romain un autre édifice fondamental, l'hippodrome de Constantinople, objet d'une seconde intervention de Jean-Claude Golvin qui en propose une restitution architecturale: cette étude s'appuie sur une thèse de doctorat soutenue à Bordeaux III par Fabricia Fauquet en 2002 (laquelle a montré par exemple que les douze carceres étaient juxtaposés sans interruption, la porta pompae étant rejetée à l'extrémité ouest), et elle bénéficie de la publication remarquable qu'a donnée Gilbert Dagron en 2000 du Livre des Cérémonies ${ }^{15}$ : on peut désormais considérer que les principales caractéristiques de l'édifice sont établies ainsi que son fonctionnement. La restitution aboutit à un dessin d'ensemble de l'hippodrome dans son contexte urbain (pl. V), un de ces dessins dont

I2. Y. Porath, J. Patrich dans le Journal of Roman Archaeology et ses suppléments chers à John Humphrey.

13. J. Heiden, Die Tondächer von Olympia, p. 105-111, fig. 21-25 et W. Decker, J.-P. Thuillier, Le sport dans l'Antiquité, p. 104 sq.

I4. D. G. Romano, "A Roman circus in Corinth».

I5. G. Dagron, «L'organisation et le déroulement des courses d'après le Livre des Cérémonies". 
Jean-Claude Golvin a le secret et qui suscitent parfois quelques critiques, mais l'auteur estime que le tout n'est pas totalement imaginaire, et qu'il vaut la peine d'affronter ce défi pour ne pas laisser l'hippodrome «seul au milieu d'un espace informe».

L'intérêt d'un colloque comme celui-là est de réunir les informations archéologiques les plus récentes. De ce point de vue, on ne sera pas déçu par la péninsule ibérique, autre terre dédiée avec passion aux courses hippiques de l'époque romaine. Le long et remarquable article de Trinidad Nogales Basarrate permet de faire le point sur les nouvelles découvertes et les publications récentes concernant les cirques, et cette étude s'accompagne de toute une série de tableaux, de cartes, de plans, de reconstitutions, de photos qui éclairent bien des informations ${ }^{16}$. J'avoue un faible pour le montage de la figure 4 qui donne le plan du cirque de Tarragone avec des vignettes montrant les différents vestiges qui apparaissent en particulier dans des restaurants: si l'on songe aussi au cas de Tolède, cela confirme qu'on trouve bien tout dans les auberges espagnoles, et en particulier donc des restes de circus! Les cirques nouvellement identifiés sur le terrain sont en Tarraconaise ceux de Segobriga - découverte récentissime puisque encore inédite ${ }^{17}$ - et de Valence, aux dimensions très semblables $(350 \mathrm{~m}$ x $70 \mathrm{~m})$ à celles du cirque de Sagonte, situé tout près; en Lusitanie, celui d'Olisipo (actuelle Lisbonne), dans la zone de la célèbre Praça do Rossio - mais les dimensions n'en sont pas établies; en Bétique est maintenant comblé le vide archéologique, que John Humphrey trouvait surprenant et en contradiction avec un dossier épigraphique très fourni : on connaît désormais deux cirques supplémentaires, d'abord à Cordoue, ce qui est bien normal, mais dans la partie orientale de la capitale provinciale, puis à Carmo, l'actuelle Carmona. Ainsi s'affirme encore plus "l'exception ibérique", avec ce nombre désormais impressionnant de cirques: seule la zone nord-ouest de la péninsule reste un peu vide de ce point de vue, mais, comme il arrive souvent, c'est peut-être seulement en raison de l'absence de fouilles et de prospections. On ne peut que féliciter encore pour cette présentation l’archéologue de Mérida, la capitale de Lusitanie qui offre à notre vue depuis longtemps un cirque si spectaculaire.

I6. J. Nelis-Clément, J.-M. Roddaz (éds), Le cirque romain et son image, p. 161-202.

17. L'information est due à la courtoisie du Prof. Juan Manuel Abascal. 
Pour la Narbonnaise, Claude Sintes nous donne une courte mais suggestive synthèse du cirque d'Arles, en rappelant qu'aucune découverte nouvelle n'a été faite dans les Gaules et dans les Germanies - alors qu'on a trouvé par exemple un théâtre à Aix et peut-être un stade à Vienne. Deux cents alvéoles, de 5,95 m sur $3 \mathrm{~m}$, devaient constituer l'ensemble de la structure porteuse et un pilotis de fondation est partout présent, qui a dû exiger environ 25000 arbres, le pin étant utilisé sous le mur de façade et le mur de podium, et le chêne sous les entretoises qui recevaient le poids des voûtes. Ici, la piste, ce qui est rare en dehors de Carthage, a pu être fouillée, avec ses couches d'argile superposées et son cailloutis de calcaire damé. En tout cas, cet extraordinaire pilotis, qui pose aussi la question des commanditaires, a permis de dater la construction du milieu du $\mathrm{II}^{\mathrm{e}}$ siècle de notre ère. Une datation précise qui est essentielle, car elle montre que l'édifice est postérieur à la reconstruction du Grand Cirque de Rome en 103 apr. J.-C., et que celui-ci a donc pu servir de modèle au monument arlésien. Il n'y a pas eu de cirque "provisoire" auparavant (vestiges d'habitat) et surtout, on sait aussi désormais que le fameux obélisque d'Arles n'est pas égyptien, mais qu'il vient de Turquie, près d'Alexandrie de Troade. Rien ne prouve d'ailleurs qu'il ait été érigé seulement sous Constantin, même si cette hypothèse est séduisante: le cirque est abandonné en tout cas après 550 , alors qu'un habitat tardif s'était installé dans certaines de ses parties dès la fin du IV siècle.

Quant au cirque de Vienne, il a été bien étudié par Anne Le Bot dont l'analyse, déjà présentée en partie lors du colloque de Lattes (1990) sur les édifices de cirque et les spectacles du cirque, n'a malheureusement pas pu figurer dans cet ouvrage. Grâce à l'étude de rapports et de plans anciens et à divers sondages, elle a pu apporter des conclusions nouvelles en particulier sur le plan chronologique ${ }^{18}$ : elle a ainsi pu mettre en évidence l'existence de deux phases et presque de deux cirques, dans la zone située à environ $500 \mathrm{~m}$ au sud de l'enceinte du Haut-Empire, le long du Rhône (ce qui est le cas aussi de celui d'Arles). Le premier monument, interprété parfois auparavant comme un xyste ou un stade, est à dater de la fin du I ${ }^{\text {er }}$ siècle de notre ère: il aurait eu des tribunes en bois et on connaît surtout la largeur de l'arène qui serait de $70 \mathrm{~m}$. Quant au second monument, en pierre évidemment et de dimensions plus importantes, il serait de la seconde moitié du $\mathrm{II}^{\mathrm{e}}$ siècle, époque où la ville connaît un

I8. A. Le Bot-Helly, B. Helly, «Les édifices de spectacle dans la Vienne romaine». 
grand développement économique; il a été l'objet de plusieurs aménagements, le dernier au IV ${ }^{\mathrm{e}}$ siècle peut-être (John Humphrey pensait même que tout l'édifice était tardif). De cet édifice, on connaît depuis toujours la barrière grâce à la fameuse Pyramide qui la surmontait (et qui a donné son nom à un célèbre restaurant gastronomique...).

En 1986, John Humphrey était obligé de conclure son chapitre sur "the evidence for chariot racing in Britain" par une phrase un peu désabusée: "...chariot racing never became an important sport in Britain " ${ }^{19}$. De fait, si on avait bien mis au jour dans la province des documents (mosaïques, céramique, verres) représentant des ludi circenses, on n'avait identifié aucun cirque monumental. Tout en évoquant la possibilité qu'on trouve un jour un cirque à Colchester, John Humphrey penchait plutôt pour Londres, où d'ailleurs l'existence de murs parallèles assez longs pouvait conforter une telle hypothèse. Mais c'est Colchester qui a décroché la timbale en 2004 avec la découverte, au sud de la colonie romaine (Colonia Victricensis), d'un cirque monumental en pierre érigé au $\mathrm{II}^{\mathrm{e}}$ siècle de notre ère: un véritable événement quand on connaît le vide des provinces romaines septentrionales sur ce point. L'article de Philip Crummy permet de cerner les dimensions de l'édifice qui a une longueur moyenne (autour de $450 \mathrm{~m}$ ) mais une largeur assez faible (autour de $72 \mathrm{~m}$ ) : il semble d'ailleurs avoir été doté de huit carceres seulement - si le système des factions était en vigueur dans la région, cela impliquerait évidemment que seules des courses singulae ou binae étaient possibles, mais une telle hypothèse reste parfaitement problématique. Si ce cirque n’a évidemment pas été dégagé en entier, divers sondages ont apporté d'intéressants renseignements qui viennent conforter sur le plan archéologique ce que l'on pouvait déjà supposer. Ainsi la mise au jour de frettes en fer a-t-elle permis de constater que des canalisations apportaient l'eau jusqu'à la barrière centrale, l'euripe - et c'est là que les sparsores venaient la puiser pour leurs amphores paillées ou leurs cuvettes; la fouille précise du sol de l'arène autour d'une des bornes confirme bien que les cochers s'approchaient le plus possible de celles-ci, et que dans ces conditions les naufrages ne devaient pas être si rares. L'édifice a dû être utilisé jusqu'à la fin du III e siècle apr. J.-C.

Les deux articles qui concluent cette première partie archéologique et avec lesquels nous arrêterons notre propre compte rendu forment une

19. J. H. Humphrey, Roman circuses, p. 437. 
annexe méthodologique. Ils sont l'œuvre de deux des protagonistes de cette grande opération Circus Maximus: Robert Vergnieux s'interroge sur l'élaboration, l'utilisation scientifique et la valorisation des modèles 3D du Circus Maximus et Jean-Claude Golvin revient sur l'exploitation des images antiques en passant par la terminologie des sciences cognitives. Larticle de Robert Vergnieux lâche une petite bombe en passant: en testant à partir du modèle 3D la capacité de l'édifice, on arriverait (calculs de P. Mora) à un chiffre moyen de... 80000 places. Ainsi, bien loin des 250000 spectateurs envisagés à une époque d'après Pline l'Ancien, et surtout encore loin des 150000 spectateurs, nombre qu'on retenait habituellement depuis les études de John Humphrey et dont la fiabilité s'appuyait aussi sur une remarque de Denys d'Halicarnasse. J'avoue que je serais très déçu s'il fallait se rallier à un chiffre aussi bas, même si c'est là la capacité du Stade de France à Saint-Denis! Attendons d'autres analyses et d'autres éléments de discussion: on voit qu'il reste encore du pain sur la planche et que - et c'est heureux - cette très belle publication ne referme pas le dossier du Grand Cirque de Rome.

Jean-Paul Thuillier

Ecole Normale Supérieure, Paris 


\section{BIBLIOGRAPHIE}

Barat, Yvan, Venet, Valerian, «Epaphrodite et sa "gazelle”: un souvenir du Circus Maximus aux confins de l'Ile-de-France", Revue Archéologique d'Ile-de-France, 1 (2008), p. 209-214.

DaGron, Gilbert, "L'organisation et le déroulement des courses d'après le Livre des Cérémonies", Travaux et Mémoires, 13 (2000), p. 1-200.

Decker, Wolfgang, Thuillier, Jean-Paul, Le sport dans l'Antiquité: Egypte, Grèce, Rome, Paris, Picard, 2004.

FAUQUeT, Fabricia, Le cirque romain, essai de théorisation de sa forme et de ses fonctions, thèse de doctorat soutenue à l'Université de Bordeaux III, 2002 (en cours de publication).

Heiden, Joachim, Die Tondücher von Olympia, Berlin/New York, W. de Gruyter, 1995.

Humphrey, John H., Roman circuses. Arenas for chariot racing, London, Batsford, 1986.

Ioppolo, Giovanni, Pisani Sartorio, Giuseppina, La villa di Massenzio sulla Via Appia: il circo, Roma, Ed. Colombo, 1999.

Le Bot-Helly, Anne, Helly, Benoît, «Les édifices de spectacle dans la Vienne romaine", in Les Allobroges. Gaulois et Romains du Rhône aux Alpes. De l'indépendance à la période romaine (4e s. av. J.-C. $-2^{e}$ s. apr. J.-C.), éd. J.-P. Jospin, Gollion, Infolio, 2002, p. 124-131.

Nelis-Clément, Jocelyne, Roddaz, Jean-Michel (éds), Le cirque romain et son image, Bordeaux, Ausonius, 2008.

Patrich, Joseph, "More on the hippodrome-stadium of Caesarea Maritima: a response to the comments of Y. Porath", JRA 16 (2003), p. 456-459.

Porath, Yosef, "Herod's circus at Caesarea: a response to J. Patrich (JRA 14, 269-283) ", JRA 16 (2003), p. 451-455.

Romano, David G., "A Roman circus in Corinth», Hesperia, 74 (2005), p. 585-611. 
Thuillier, Jean-Paul, «Agitator ou sparsor? A propos d'une célèbre statue de Carthage", Comptes Rendus des séances de l'Académie des Inscriptions et Belles Lettres, (1999), p. 1081-1106.

Tremel, Jan, Magica agonistica. Fluchtafeln im antiken Sport, Hildesheim, Weidman, 2004.

WeLCH, Katherine E., The Roman amphitheatre: from its origins to the Colosseum, Cambridge, Cambridge University Press, 2007. 
